\title{
A Practical Risk Score to Predict 24-Month Post-Discharge Mortality Risk in Patients With Non-ST-Segment Elevation Myocardial Infarction
}

\author{
Rui Fu, MD; Chenxi Song, MD; Jingang Yang, MD, PhD; Chuanyu Gao, MD, PhD; \\ Yan Wang, MD, PhD; Haiyan Xu, MD, PhD; Xiaojin Gao, MD; Xiaoxue Fan, MD; \\ Han Xu, MD; Hao Wang, MD; Kefei Dou, MD, PhD; \\ Yuejin Yang, MD, PhD on behalf of the CAMI Registry Study
}

\begin{abstract}
Background: Risk stratification of patients with non-ST-segment elevation myocardial infarction (NSTEMI) is important in terms of treatment strategy selection. Current efforts have focused on short-term risk prediction after discharge, but we aimed to establish a risk score to predict the 24-month mortality risk in survivors of NSTEMI.
\end{abstract}

\begin{abstract}
Methods and Results: A total of 5,509 patients diagnosed with NSTEMI between January 2013 and September 2014 were included. Primary endpoint was all-cause death at 24 months. A multivariable Cox regression model was used to establish a practical risk score based on independent risk factors of death. The risk score included 9 variables: age, body mass index, left ventricular ejection fraction, reperfusion therapy during hospitalization, Killip classification, prescription of diuretics at discharge, heart rate, and hemoglobin and creatinine levels. The C-statistics for the risk model were 0.83 (95\% confidence interval [Cl]: 0.81-0.85) and 0.83 (95\% Cl: 0.79-0.86) in the development and validation cohorts, respectively. Mortality risk increased significantly across groups: $1.34 \%$ in the low-risk group (score: 0-58), 5.40\% in intermediate group (score: 59-93), and 23.87\% in high-risk group (score: $\geq 94$ ).

Conclusions: The current study established and validated a practical risk score based on 9 variables to predict 24-month mortality risk in patients who survive NSTEMI. This score could help identify patients who are at high risk for future adverse events who may benefit from good adherence to guideline-recommended secondary prevention treatment.
\end{abstract}

Key Words: Long-term outcome; Non-ST elevation myocardial infarction; Risk prediction model

$\mathbf{T}$ he number of patients surviving acute myocardial infarction (AMI) has increased in recent years ${ }^{1}$ due to advances in early diagnosis and improved treatment. ${ }^{2}$ However, survivors of AMI have varying risk of recurrent cardiovascular events. ${ }^{2}$ AMI is most commonly classified as ST-elevation MI (STEMI) or non-STEMI (NSTEMI) based on ECG characteristics. Compared with patients with STEMI, those with NSTEMI display worse long-term clinical outcomes. ${ }^{3}$ Accurate risk stratification, particular among patients with NSTEMI, may assist in identifying those at high risk of future adverse cardiac events and who may benefit from intensive secondary prevention to improve outcomes.

Several scores have been developed to predict prognosis in the context of acute coronary syndrome (ACS), including TIMI score, ${ }^{4}$ GRACE score, ${ }^{5}$ ACTION score, ${ }^{6}$ Canada Acute Coronary Syndrome risk score, ${ }^{7}$ and TRS-2P score. ${ }^{8}$ These scores focus on risk prediction in the acute phase of AMI (i.e., the risk of death within the first year after discharge). However, patients who survive AMI still have a high risk of adverse cardiovascular events beyond the first year. ${ }^{9}$ The objective of our study was to develop and validate a risk score to predict long-term risk in patients who survive NSTEMI.

CAMI Registry

\section{Methods}

A detailed description of the CAMI registry was reported

Received May 25, 2020; revised manuscript received July 7, 2020; accepted July 15, 2020; J-STAGE Advance Publication released online September 17, 2020 Time for primary review: 21 days

Fuwai Hospital, National Center for Cardiovascular Diseases, Chinese Academy of Medical Science and Peking Union Medical College, Beijing (R.F., C.S., J.Y., Haiyan X., X.G., X.F., Han X., H.W., K.D., Y.Y.); Department of Cardiology, Henan Provincial People's Hospital, Fuwai Central China Cardiovascular Hospital, People's Hospital of Zhengzhou University, Zhengzhou, Henan (C.G.); and Xiamen Cardiovascular Hospital Xiamen University, Xiamen (Y.W.), China

K.D. and Y.Y. contributed equally and accept equal and full responsibility for the work as correspondence authors.

Mailing address: Yuejin Yang, MD, PhD and Kefei Dou, MD, PhD, Department of Cardiology, Fuwai Hospital, 167 Beilishi Road, Beijing 100037, People's Republic of China. E-mail: yangyjfw@126.com or drdoukefei@126.com

All rights are reserved to the Japanese Circulation Society. For permissions, please e-mail: cj@j-circ.or.jp

ISSN-1346-9843 


\begin{tabular}{|c|c|c|c|}
\hline Variable & $\begin{array}{l}\text { Patients died } \\
\quad(n=584)\end{array}$ & $\begin{array}{l}\text { Patients alive } \\
(n=4,925)\end{array}$ & $P$ value \\
\hline Age (years) & $73.59 \pm 9.63$ & $63.95 \pm 11.78$ & $<0.001$ \\
\hline Female & $244 / 584(41.8 \%)$ & $1,448 / 4,925(29.4 \%)$ & $<0.001$ \\
\hline BMI $\left(\mathrm{kg} / \mathrm{m}^{2}\right)$ & $23.19 \pm 3.04$ & $24.18 \pm 3.03$ & $<0.001$ \\
\hline Diabetes & $186 / 583(31.9 \%)$ & $1,094 / 4,925(29.4 \%)$ & $<0.001$ \\
\hline Hypertension & $381 / 584(65.2 \%)$ & $2,813 / 4,900(57.4 \%)$ & $<0.001$ \\
\hline Hyperlipidemia & $36 / 584(6.2 \%)$ & 430/4,898 (8.8\%) & 0.025 \\
\hline LVEF (\%) & $47.65 \pm 12.73$ & $55.82 \pm 11.39$ & $<0.001$ \\
\hline \multicolumn{4}{|l|}{ Past medical history } \\
\hline MI & $103 / 584(17.6 \%)$ & $489 / 4,887(10 \%)$ & $<0.001$ \\
\hline HF & $80 / 584(13.7 \%)$ & $189 / 4,888(3.9 \%)$ & $<0.001$ \\
\hline $\mathrm{PCl}$ & $31 / 584(5.3 \%)$ & $250 / 4,874(5.1 \%)$ & 0.856 \\
\hline CABG & $8 / 583(1.4 \%)$ & $41 / 4,888(0.8 \%)$ & 0.475 \\
\hline Stroke & $98 / 584(16.8 \%)$ & $452 / 4,886(9.3 \%)$ & $<0.001$ \\
\hline Renal failure & $38 / 584(6.5 \%)$ & $99 / 4,876(2.0 \%)$ & $<0.001$ \\
\hline COPD & $36 / 582(6.2 \%)$ & $98 / 4,861(2.0 \%)$ & $<0.001$ \\
\hline Family history of premature CAD & $4 / 584(0.7 \%)$ & $164 / 4,894(3.4 \%)$ & $<0.001$ \\
\hline PAD & $14 / 584(2.4 \%)$ & $51 / 4,882(1.0 \%)$ & 0.039 \\
\hline Smoking status & & & $<0.001$ \\
\hline Non-smoker & $358 / 584(61.3 \%)$ & $2,400 / 4,883(49.2 \%)$ & \\
\hline Ex-smoker & $110 / 584(18.8 \%)$ & $621 / 4,883(12.7 \%)$ & \\
\hline Current smoker & $116 / 584(19.9 \%)$ & $1,862 / 4,883(38.1 \%)$ & \\
\hline Killip classification & & & $<0.001$ \\
\hline I & $256 / 581(44.1 \%)$ & $3666 / 4,879(75.1 \%)$ & \\
\hline II & $160 / 581(27.5 \%)$ & $837 / 4,879(17.2 \%)$ & \\
\hline III & $118 / 581(20.3 \%)$ & $269 / 4,879(5.5 \%)$ & \\
\hline IV & $47 / 581(8.1 \%)$ & $107 / 4,879(2.2 \%)$ & \\
\hline Cardiac arrest & $3 / 583(0.5 \%)$ & $26 / 4,886(0.5 \%)$ & 1.000 \\
\hline Primary PCl & $24 / 572(4.2 \%)$ & $506 / 4,814(10.5 \%)$ & $<0.001$ \\
\hline \multicolumn{4}{|l|}{ Discharge medications } \\
\hline Aspirin & $465 / 546(85.2 \%)$ & $4,386 / 4,771(91.9 \%)$ & $<0.001$ \\
\hline Clopidogrel & $443 / 546(81.1 \%)$ & $4,165 / 4,771(87.3 \%)$ & $<0.001$ \\
\hline Statins & $471 / 546(86.3 \%)$ & $4,398 / 4,771(92.2 \%)$ & $<0.001$ \\
\hline$\beta$-blockers & $317 / 546(58.1 \%)$ & $3,300 / 4,771(69.2 \%)$ & $<0.001$ \\
\hline ACEI/ARB & $315 / 546(57.7 \%)$ & $2,944 / 4,771(61.7 \%)$ & 0.069 \\
\hline
\end{tabular}

ACEI, angiotensin-converting enzyme inhibitor; ARB, angiotensin II receptor blocker; BMI, body mass index; CABG, coronary artery bypass graft; CAD, coronary artery disease; COPD, chronic obstructive pulmonary disease; HF, heart failure; LVEF, left ventricular ejection fraction; MI, myocardial infarction; PAD, Peripheral artery disease; PCI, percutaneous coronary intervention.

previously. ${ }^{10}$ Briefly, it was a multicenter prospective registry enrolling Chinese patients with AMI. Eligible patients were admitted within 7 days of acute ischemic symptoms with a primary diagnosis of AMI, including STEMI and NSTEMI. A total of 108 hospitals of 3 administrative levels (province-, prefecture- or country-level) from 31 provinces and 4 municipalities participated in the registry, which assured good representation of Chinese patients with AMI. The CAMI registry was approved by the Institutional Review Board Central Committee at Fuwai Hospital, National Center for Cardiovascular Diseases, China (No. 2012-431). All procedures were conducted in accordance with the "Declaration of Helsinki" and the ethical standards of the responsible committee. Written informed consent was given by every eligible patients before registration. CAMI is registered on www.clinicaltrials.gov (No. NCT01874691).

\section{Study Population}

The present study included patients diagnosed with NSTEMI, which was defined in accordance with Third Universal Definition of Myocardial Infarction: that is, a rise or fall of cardiac biomarkers with at least 1 value above the 99th percentile upper reference limit and at least one of the following: (1) symptoms of ischemia; (2) absence of ST-segment elevation on ECG; (3) imaging evidence of new loss of viable myocardium or new regional wall motion abnormality; and (4) identification of an intracoronary thrombus by angiography or autopsy. Patients with missing or unavailable data on age, body mass index (BMI), and left bundle branch block (LBBB), missing data on 24-month follow-up and those who died during hospitalization were excluded.

\section{Data Collection and Definition}

Data were collected by trained staff using a standardized 


\begin{tabular}{lcc|}
\hline \multicolumn{1}{c|}{ Table 2. Independent Predictors of Mortality at 24-Month Follow-up } & P value \\
Age (per 1 year increase) & HR (95\% Cl) & $<0.001$ \\
BMI (per $1 \mathrm{~kg} / \mathrm{m}^{2}$ increase) & $1.050(1.040,1.059)$ & 0.002 \\
Previous MI history & $0.957(0.930,0.984)$ & 0.002 \\
Previous stroke history & $1.412(1.136,1.754)$ & 0.010 \\
No in-hospital revascularization & $1.335(1.072,1.663)$ & $<0.001$ \\
Killip classification (per 1 classification increase) & $2.295(1.825,2.886)$ & $<0.001$ \\
ST-segment depression & $1.241(1.134,1.359)$ & 0.044 \\
Statins at discharge & $1.191(1.005,1.412)$ & 0.002 \\
Diuretics at discharge & $0.665(0.516,0.856)$ & 0.034 \\
LVEF\% (per $1 \%$ increase) & $1.218(1.015,1.461)$ & $<0.001$ \\
Heart rate (per 1 beat/min increase) & $0.979(0.972,0.985)$ & $<0.001$ \\
Hemoglobin (per $1 \mathrm{~g} / \mathrm{L}$ increase) & $1.006(1.003,1.010)$ & $<0.001$ \\
WBC count (per 10\%/L increase) & $0.994(0.991,0.998)$ & 0.002 \\
Creatinine (per $1 \mu$ mol/L increase) & $1.032(1.012,1.052)$ & $<0.001$ \\
Serum potassium (per 1 mmol/L increase) & $1.002(1.001,1.003)$ & 0.012 \\
\hline
\end{tabular}

$\mathrm{Cl}$, confidence interval; $\mathrm{HR}$, hazard ratio; WBC, white blood cell. Other abbreviations as in Table 1.

set of variables. Patients' demographics, clinical presentations, medical histories, risk factors, diagnosis and treatment, and follow-up data were collected. Definitions of variables were in accordance with the ACC/AHA Task Force on Clinical Data Standards and the NCDRACTION-GWTG element dictionary. ${ }^{10}$ The primary endpoint was all-cause death at 24-month follow-up.

\section{Patient Follow-up}

Patients were followed up at 30 days, 6 months and 24 months by telephone call or clinical visit. Data on adverse events (death, cause of death, cardiovascular events, bleeding, etc.), and medication adherence or discontinuation were collected. All clinical events were validated by source document.

\section{Statistical Analysis}

Continuous data are presented as mean $\pm \mathrm{SD}$ and compared between groups by Student's t-test. Categorical variables are presented as numbers (percentages), and compared between groups using the likelihood-ratio chi-square or the Fisher exact test if appropriate.

\section{Risk Score Development}

The entire study population was divided into 2 cohorts chronologically: a derivation cohort of 4,132 patients to develop the risk model and a validation cohort of 1,377 patients to test and validate the risk model. A univariable Cox regression analysis was first performed to assess the association between each baseline variable and 24-month death risk. Variables with $\mathrm{P}<0.05$ were selected to fit a backward multivariate Cox model. The variables with $\mathrm{P}<0.05$ were used to construct the final multivariate Cox model. We then created a simple risk score that is easy to use in clinical practice. The variable with the smallest coefficient was selected as the reference variable and attributed 1 point. The scores of the other variables were determined by dividing their estimated coefficients by the coefficient of the reference variable. The variance inflation factor was calculated to assess multicollinearity between variables. Area under the curve (AUC) value and the HosmerLemeshow (HL) goodness-of-fit test were used to assess the discrimination and calibration ability of the model. The AUC value, net reclassification improvement and the integrated discriminatory index were calculated to compare diagnostic performance between different models. All analyses were performed with SAS 9.4 (SAS Institute, Cary, NC, USA).

\section{Results}

\section{Baseline Characteristics}

A total of 6,327 patients with NSTEMI were registered from January 2013 to September 2014. We excluded patients with missing or unavailable data on age $(n=120)$, BMI $(n=299)$, and LBBB $(n=42)$, and missing data on 24-month follow-up $(n=9)$. We also excluded in-hospital deaths $(n=348)$ and finally included 5,509 patients whose baseline characteristics are shown in Table 1. At 24-month follow-up, a total of 584 patients had died. Compared with patients who were alive at follow-up, those who died were older, more likely to be female and had lower BMI. The proportion of risk factors (diabetes, hypertension), and past medical history (previous history of MI, heart failure, stroke, renal failure, chronic obstructive pulmonary disease, peripheral artery disease) was higher in the patients who died at follow-up. Patients who died had higher Killip classification and lower rate of primary PCI than patients who survived (Table 1). Specific causes of death are shown in Supplementary Table 1.

\section{Independent Predictors of Mortality}

Results of the univariable Cox regression model are summarized in Supplementary Table 2. Variables with $\mathrm{P}<0.05$ were selected to enter the multivariable stepwise Cox regression model. Independent predictors of death are presented in Table 2.

\section{CAMI-NSTEMI 24-Month Post-Discharge Risk Score}

The following variables were selected to build the risk model: age, BMI, revascularization during hospitalization, Killip classification, diuretics at discharge, $\mathrm{LVEF} \%$, heart rate, and hemoglobin and creatinine levels. Scores attributed to each variable are shown in Table 3. Within the 


\begin{tabular}{|c|c|c|c|c|c|}
\hline Variable & Category & Score & Variable & Category & Score \\
\hline \multirow[t]{4}{*}{ Age (years) } & $<55$ & 0 & \multirow[t]{4}{*}{ LVEF (\%) } & $<50$ & 21 \\
\hline & {$[55-65]$} & 21 & & [50-55] & 11 \\
\hline & [65-75] & 37 & & [55-60] & 7 \\
\hline & $\geq 75$ & 53 & & $\geq 60$ & 0 \\
\hline \multirow[t]{4}{*}{ BMI $\left(\mathrm{kg} / \mathrm{m}^{2}\right)$} & $<22$ & 11 & \multirow[t]{4}{*}{ Heart rate (beat/min) } & $<65$ & 0 \\
\hline & [22-24] & 7 & & [65-75] & 4 \\
\hline & [24-26] & 5 & & [75-85] & 6 \\
\hline & $\geq 26$ & 0 & & $\geq 85$ & 13 \\
\hline \multirow{2}{*}{$\begin{array}{l}\text { Revascularization during } \\
\text { hospitalization }\end{array}$} & Yes & 0 & \multirow[t]{4}{*}{ Hemoglobin (g/L) } & $<121$ & 10 \\
\hline & No & 28 & & [121-133] & 6 \\
\hline \multirow[t]{4}{*}{ Killip classification } & 1 & 0 & & [133-145] & 4 \\
\hline & II & 9 & & $\geq 145$ & 0 \\
\hline & III & 18 & \multirow[t]{4}{*}{ Creatinine (umol/L) } & $<64$ & 0 \\
\hline & IV & 27 & & [64-76] & 2 \\
\hline \multirow[t]{2}{*}{ Diuretics at discharge } & No & 0 & & [76-93] & 3 \\
\hline & Yes & 7 & & $\geq 93$ & 9 \\
\hline
\end{tabular}

BMI, body mass index; LVEF, left ventricular ejection fraction.
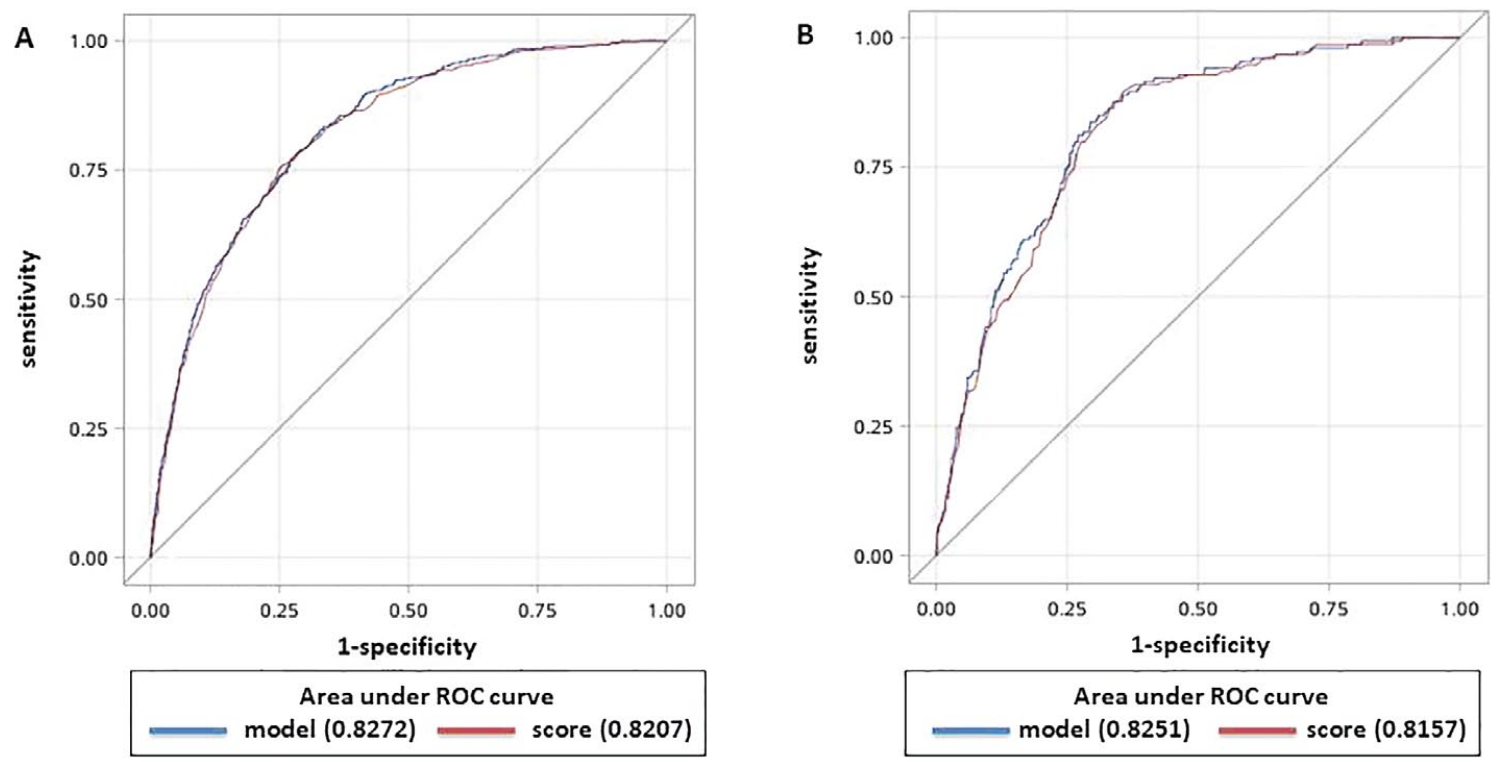

Figure 1. ROC curves of CAMI risk model and CAMI risk score. (A) Within the derivation cohort, the C-statistic was 0.83 (95\% confidence interval (CI): 0.81-0.85) for the CAMI risk model and $0.82(95 \% \mathrm{Cl}$ : 0.80-0.84) for the risk score. (B) Within the validation cohort, the C-statistic was 0.83 (95\% Cl: 0.79-0.86) for the CAMl risk model and 0.82 (95\% Cl: 0.78-0.85) for the risk score. CAMI, China Acute Myocardial Infarction; ROC, receiver-operating characteristic.

derivation cohort, the respective AUC values of the CAMI-NSTEMI risk model and risk score were $0.83(95 \%$ confidence interval (CI): $0.81-0.85)$ and $0.82(95 \% \mathrm{CI}$ : $0.80-0.84)$, and no significant difference was found $(\mathrm{P}=0.05$, Figure 1A). The respective HL test $\mathrm{P}$ values for the CAMI risk score and risk model were 0.97 and 0.63 , which indicated good calibration performance. Within the validation cohort, the respective AUC values of the CAMI-NSTEMI risk model and risk score were $0.83(95 \%$ CI: 0.79-0.86) and 0.82 (95\% CI: 0.78-0.85), and no significant difference was found $(\mathrm{P}=0.20$, Figure 1B). The respective $\mathrm{HL}$ test $\mathrm{P}$ values for risk score and model were 0.18 and 0.03 . Within the entire cohort, the AUC value of the CAMI risk score was greater than that of the GRACE risk score ( 0.82 vs. $0.78, \mathrm{P}<0.001$ for comparison, Figure 2 ).

The risk attributed to each score is shown in Table 3. To define the 3 different risk groups (low, intermediate, highrisk groups), the risk score was divided according to tertiles. Score range and event rates are shown in Table 4. The mortality rate increased significantly across the different groups (Table 4). 


\section{Discussion}

\section{Major Findings}

Using data from the CAMI registry, the present study identified 9 independent risk factors that were used to develop and validate a risk score to predict 24-month mortality risk after AMI discharge. These variables are easy to collect in routine clinical practice: age, BMI, heart rate, Killip classification, $\mathrm{LVEF}^{\circ}$, hemoglobin and creatinine levels, diuretics at discharge and revascularization during hospitalization. Our risk score showed excellent diagnostic performance and classified patients into 3 risk categories, which may assist clinicians to identify high-risk patients and select the optimal treatment for patients with AMI following discharge.

\section{Existing Risk Scores}

Many risk scores have been developed to assess mortality risk in patients with ACS, including the GRACE risk score, ${ }^{5}$ TIMI risk score ${ }^{4}$ and ACTION score, ${ }^{6}$ etc. The GRACE risk score is one of the most widely used and

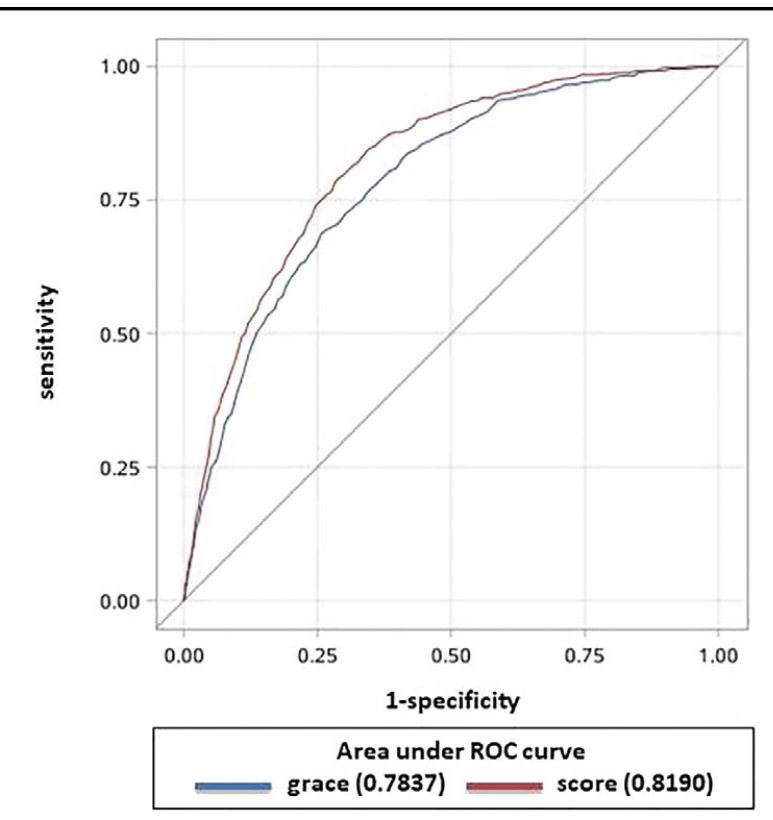

Figure 2. ROC curves for CAMI risk score and GRACE risk score. The area under the curve value (AUC) for the CAMlNSTEMI score is significantly higher than that of the GRACE score (AUC value: 0.82 vs. $0.78, \mathrm{P}<0.0001$ for comparison). CAMI, China Acute Myocardial Infarction; NSTEMI, non-STsegment elevation myocardial infarction; ROC, receiver-operating characteristic. validated scores, and was derived from the multicenter GRACE registry to estimate in-hospital and 6-month mortality risk in patients with ACS. The GRACE 6-month post-charge score includes the following 9 variables: age, history of MI, history of heart failure, pulse rate at presentation, systolic blood pressure, levels of serum creatinine and serum cardiac biomarkers, ST-segment depression on presenting ECG, and not having a percutaneous coronary intervention performed in hospital. The investigators later updated and improved the original GRACE score and developed GRACE 2.0, which is more suitable for emergency settings because it allows substitution of diuretic usage for Killip class, and substitution of history of renal dysfunction for serum creatinine. GRACE 2.0 is also more accurate than the original score because it used non-linear function.

\section{Rationale for Novel Risk Score}

Our risk score differed from the other risk scores in 3 aspects. First, our risk score evaluated long-term outcomes in patients who survive NSTEMI. Most risk scores focus on mortality risk during hospitalization or within 6 months of discharge. With more effective medication therapy and timely revascularization, the proportion of patients who survive AMI has increased, ${ }^{11}$ but because these patients are still at high risk of future adverse events, it is important to further identify independent risk factors from a long-term perspective. Second, our risk score focused on patients with NSTEMI. Accurate stratification of patients with NSTEMI is necessary because NSTEMI accounts for approximately $70 \%$ of all AMI and patients with NSTEMI have a varying prognosis. To our knowledge, no risk score to date has been developed particularly for patients with NSTEMI. Third, the present study assessed long-term prognostic factors in Asian patients post AMI. Most of the other scores are developed from populations in Europe, America, and Australia. Asian populations account for more than $60 \%$ of the population worldwide, and ACS is one of the leading cause of death in Asia and accounts for more than $50 \%$ of the disease burden.12 Therefore, it is necessary to explore independent risk factors post-AMI in Asian populations.

\section{Risk Factors}

Many of the included risk factors are consistent with those in other risk scores, including age, revascularization during hospitalization, heart rate, and systolic blood pressure, etc. Compared with them, our risk model incorporated several new variables, including hemoglobin level, and BMI. Our study found that reduced hemoglobin level was associated with increased 24-month mortality. Consistent with that finding, previous studies showed that the presence and severity of anemia was adversely associated with shortand long-term mortality risk. ${ }^{13-15}$ Compared with the gen-

\begin{tabular}{|c|c|c|c|c|}
\hline & Low-risk group & Intermediate-risk group & High-risk group & $P$ value \\
\hline Score range & $0-58$ & $59-93$ & $\geq 94$ & \\
\hline \multicolumn{5}{|l|}{ Mortality rate } \\
\hline Derivation cohort & $18 / 1,346(1.34)$ & $74 / 1,370(5.40)$ & $338 / 1,416(23.87)$ & $<0.001$ \\
\hline Validation cohort & $6 / 451(1.33)$ & $25 / 456(5.48)$ & $123 / 470(26.17)$ & $<0.001$ \\
\hline
\end{tabular}

Data are presented as number of deaths/total number of patients (percentage) within each risk group. 
eral population, the prevalence of anemia is high among patients hospitalized with AMI. ${ }^{16}$ Furthermore, reduced hemoglobin level may impair recovery post-AMI due to reduced oxygen delivered to the myocardium and subsequent increased cardiac output. However, further studies are required to explore the effect of the magnitude of anemia on mortality risk because therapy aimed at regulating hemoglobin level has failed to demonstrate clinical benefit. ${ }^{17}$

Our study showed that increased BMI was associated with lower mortality risk. Although obesity is a well-established risk factor for cardiovascular disease, overweight or obese patients with AMI had lower short- and long-term mortality risk than normal weight patients, a phenomenon known as the "obesity paradox" ${ }^{18}$ Possible mechanisms have been proposed to explain the obesity paradox; compared with normal weight patients, overweight or obese patients have greater nutritional reserve to meet the sharply increased metabolic demands during AMI. ${ }^{19}$ In addition, our previous work indicated that patients with higher BMI were younger and more likely to receive aggressive treatment, which may explain their better prognosis. ${ }^{20}$

The association between hemoglobin level, BMI and mortality risk after AMI deserves the particular attention of Chinese physicians. Regarding hemoglobin, although the adverse effect of low hemoglobin level is well-established, the prevalence of anemia is significantly higher in low- and middle-income countries, including China, compared with high-income countries. ${ }^{21}$ Regarding BMI, the association between BMI and body composition and health outcomes may differ between Asian and European or American populations. Asians generally have a higher percentage of body fat than Europeans with the same BMI, and may develop diabetes at a lower BMI.22 Another previous study including more than 1.1 million Asians found that the shape of the curve for the BMI-mortality association was different between Asian and European populations. ${ }^{23}$

Low hemoglobin and BMI may also increase mortality risk via increased bleeding risk. Both of these factors were independent risk factors for bleeding after PCI with drugeluting stents. ${ }^{24}$ Bleeding, particularly major bleeding, is a well-established risk factor for death. In addition, bleeding may cause reduced use of antiplatelet drugs, which also contributes to worse prognosis.

More attention is required to improve adherence to optimal guideline-indicated secondary prevention treatment, which can be summarized as the following: discharge medications include aspirin, P2Y12 inhibitor, angiotensin-converting enzyme inhibitor/angiotensin-receptor blocker, $\beta$-blocker and statins, referral for cardiac rehabilitation, and advice on diet and smoking cessation..$^{25}$ However, previous studies report that the proportion of patients receiving optimal care decreased with GRACE risk score, and only $11.5 \%$ high-risk patients received all eligible treatments. ${ }^{26}$

\section{Study Limitations}

Our risk score was developed and validated in a single cohort. Although this method has been used in many studies, ${ }^{27}$ our risk score still requires further external validation in other independent cohorts. Second, cardiac troponin T (cTn) level is an important prognostic factor in patients with AMI. However, cTn was not included in our risk score because the CAMI registry was a multicenter registry with 108 participating hospitals. The method used for $\mathrm{cTn}$ measurement and cTn normal range differed across hospi- tals. To enable wide application of our risk score, we did not include cTn level as a variable.

\section{Conclusions}

We developed and validated a 9-variable risk score to predict 24-month mortality risk in patients with NSTEMI. Our risk model may assist clinicians to better identify high-risk patients and guide decision making for AMI treatment following discharge.

\section{Acknowledgments}

We thank all the members of the Scientific Committee and Executive and Steering Committee for their contribution to the CAMI registry.

This work was supported by the CAMS Innovation Fund for Medical Sciences (CIFMS) (2016-I2M-1-009), the Twelfth Five-Year Planning Project of the Scientific and Technological Department of China (2011BAI11B02), National key R\&D program of China (2018YFC1315602) and Fundamental Research Funds for the Central Universities (2018-F04).

\section{Conflict of Interest}

All authors declare no conflicts of interest.

\section{Data Availability Statement}

Data are available on reasonable request.

\section{Ethics}

The current study was approved by the Institutional Review Board Central Committee at Fuwai Hospital, National Center for Cardiovascular Diseases, China (No. 2012-431).

\section{Disclosures}

All authors declare no conflicts of interest.

\section{References}

1. Tisminetzky M, Wang TY, Gurwitz J, Kaltenbach LA, McManus $\mathrm{D}$, Gore $\mathrm{J}$, et al. Magnitude and characteristics of patients who survived an acute myocardial infarction. J Am Heart Assoc 2017; 6: $\mathrm{e} 006373$.

2. Johansson S, Rosengren A, Young K, Jennings E. Mortality and morbidity trends after the first year in survivors of acute myocardial infarction: A systematic review. BMC Cardiovasc Disord 2017; 17: 53.

3. Park HW, Yoon CH, Kang SH, Choi DJ, Kim HS, Cho MC, et al. Early- and late-term clinical outcome and their predictors in patients with ST-segment elevation myocardial infarction and non-ST-segment elevation myocardial infarction. Int $J$ Cardiol 2013; 169: 254-261.

4. Antman EM, Cohen M, Bernink PJ, McCabe CH, Horacek T, Papuchis G, et al. The TIMI risk score for unstable angina/nonST elevation MI: A method for prognostication and therapeutic decision making. JAMA 2000; 284: 835-842.

5. Eagle KA, Lim MJ, Dabbous OH, Pieper KS, Goldberg RJ, Van de Werf $\mathrm{F}$, et al. A validated prediction model for all forms of acute coronary syndrome: Estimating the risk of 6-month postdischarge death in an international registry. JAMA 2004; 291: $2727-2733$

6. McNamara RL, Kennedy KF, Cohen DJ, Diercks DB, Moscucci $\mathrm{M}$, Ramee S, et al. Predicting in-hospital mortality in patients with acute myocardial infarction. J Am Coll Cardiol 2016; 68: $626-635$.

7. Huynh T, Kouz S, Yan AT, Danchin N, O'Loughlin J, Schampaert E, et al. Canada Acute Coronary Syndrome Risk Score: A new risk score for early prognostication in acute coronary syndromes. Am Heart J 2013; 166: 58-63.

8. Bohula EA, Bonaca MP, Braunwald E, Aylward PE, Corbalan R, De Ferrari GM, et al. Atherothrombotic risk stratification and the efficacy and safety of vorapaxar in patients with stable ischemic heart disease and previous myocardial infarction. Circulation 2016; 134: 304-313.

9. Jernberg T, Hasvold P, Henriksson M, Hjelm H, Thuresson M, 
Janzon M. Cardiovascular risk in post-myocardial infarction patients: Nationwide real world data demonstrate the importance of a long-term perspective. Eur Heart J 2015; 36: $1163-$ 1170.

10. Xu H, Li W, Yang J, Wiviott SD, Sabatine MS, Peterson ED, et al. The China Acute Myocardial Infarction (CAMI) Registry: A national long-term registry-research-education integrated platform for exploring acute myocardial infarction in China. Am Heart J 2016; 175: 193-201.e3.

11. Reed GW, Rossi JE, Cannon CP. Acute myocardial infarction. Lancet 2017; 389: 197-210.

12. Ohira T, Iso H. Cardiovascular disease epidemiology in Asia: An overview. Circ J 2013; 77: 1646-1652.

13. Bernhardt P, Walcher T, Rottbauer W, Wöhrle J. Quantification of myocardial perfusion reserve at 1.5 and 3.0 Tesla: A comparison to fractional flow reserve. Int J Cardiovasc Imaging 2012; 28: $2049-2056$

14. Aronson D, Suleiman M, Agmon Y, Suleiman A, Blich M, Kapeliovich $\mathrm{M}$, et al. Changes in haemoglobin levels during hospital course and long-term outcome after acute myocardial infarction. Eur Heart J 2007; 28: 1289-1296.

15. Colombo MG, Kirchberger I, Amann U, Heier M, Thilo C, Kuch B, et al. Association between admission anemia and longterm mortality in patients with acute myocardial infarction: Results from the MONICA/KORA myocardial infarction registry. BMC Cardiovasc Disord 2018; 18: 50.

16. Bolinska S, Sobkowicz B, Zaniewska J, Chlebinska I, Bolinski J, Milewski R, et al. The significance of anaemia in patients with acute ST-elevation myocardial infarction undergoing primary percutaneous coronary intervention. Kardiol Pol 2011; 69: $33-39$.

17. Steppich B, Groha P, Ibrahim T, Schunkert H, Laugwitz KL, Hadamitzky M, et al. Effect of erythropoietin in patients with acute myocardial infarction: Five-year results of the REVIVAL-3 trial. BMC Cardiovasc Disord 2017; 17: 38.

18. Elagizi A, Kachur S, Lavie CJ, Carbone S, Pandey A, Ortega FB, et al. An overview and update on obesity and the obesity paradox in cardiovascular diseases. Prog Cardiovasc Dis 2018; 61: $142-150$.
19. Niedziela J, Hudzik B, Niedziela N, Gasior M, Gierlotka M, Wasilewski J, et al. The obesity paradox in acute coronary syndrome: A meta-analysis. Eur J Epidemiol 2014; 29: 801-812.

20. Song C, Fu R, Yang J, Xu H, Gao X, Feng L, et al. The association between body mass index and in-hospital outcome among patients with acute myocardial infarction: Insights from China Acute Myocardial Infarction (CAMI) registry. Nutr Metab Cardiovasc Dis 2019; 29: 808-814.

21. Balarajan Y, Ramakrishnan U, Ozaltin E, Shankar AH, Subramanian SV. Anaemia in low-income and middle-income countries. Lancet 2011; 378: 2123-2135.

22. WHO Expert Consultation. Appropriate body-mass index for Asian populations and its implications for policy and intervention strategies. Lancet 2004; 363: 157-163.

23. Zheng W, McLerran DF, Rolland B, Zhang X, Inoue M, Matsuo $\mathrm{K}$, et al. Association between body-mass index and risk of death in more than 1 million Asians. N Engl J Med 2011; 364: 719729.

24. Baber U, Mehran R, Giustino G, Cohen DJ, Henry TD, Sartori $\mathrm{S}$, et al. Coronary thrombosis and major bleeding after PCI with drug-eluting stents: Risk scores from PARIS. J Am Coll Cardiol 2016; 67: 2224-2234.

25. Dondo TB, Hall M, Timmis AD, Gilthorpe MS, Alabas OA, Batin PD, et al. Excess mortality and guideline-indicated care following non-ST-elevation myocardial infarction. Eur Heart $J$ Acute Cardiovasc Care 2017; 6: 412-420.

26. Hall M, Bebb OJ, Dondo TB, Yan AT, Goodman SG, Bueno H, et al. Guideline-indicated treatments and diagnostics, GRACE risk score, and survival for non-ST elevation myocardial infarction. Eur Heart J 2018; 39: 3798-3806.

27. Wang Y, Li J, Zheng X, Jiang Z, Hu S, Wadhera RK, et al. Risk factors associated with major cardiovascular events 1 year after acute myocardial infarction. JAMA Netw Open 2018; 1: e181079.

\section{Supplementary Files}

Please find supplementary file(s);

http://dx.doi.org/10.1253/circj.CJ-20-0509 